\title{
A DIMENSIONAL COMPARISON OF A SELF-REPORT AND A STRUCTURED INTERVIEW MEASURE OF CONDUCT DISORDER
}

\author{
Shannon E. Kelley, BA, Steve Balsis, PhD, Shannon Toney Smith, MA, \\ John F. Edens, PhD, Kevin S. Douglas, LLB, PhD, and Norman G. \\ Poythress, Jr., PhD
}

\begin{abstract}
Eligibility for a diagnosis of antisocial personality disorder (ASPD) requires evidence of antecedent conduct disorder (CD). Accurately identifying CD may be influenced by various factors, including assessment methodology. The present study used a two-parameter latent variable model to examine the relative performance of a self-report measure and a structured clinical interview in retrospectively detecting the CD spectrum among adult male offenders $(N=1,159)$. Self-report and clinical interview tended to converge regarding the rank order of severity indicated by $\mathrm{CD}$ symptom criteria. In addition, at relatively low levels of CD severity, self-report provided more information about the $\mathrm{CD}$ spectrum than did clinical interview. At relatively higher levels of CD severity, however, clinical interview provided more information about the CD spectrum than did self-report. Latent variable models offer a potential means of combining multiple assessment methods in a way that maximizes information gleaned by capitalizing on the contextual strengths of each approach.
\end{abstract}

The assessment of antisocial personality disorder (ASPD) symptoms is useful to inform decision making in the supervision of clinical and forensic populations, including appropriate intervention selection and risk-reduction efforts (Black, Gunter, Loveless, Allen, \& Sieleni, 2010). However, a formal diagnosis of ASPD requires retrospective evidence of conduct disorder (CD) with onset

From Texas A\&M University (S. E. K., S. B., S. T. S., J. F. E.); Simon Fraser University and Mid-Sweden University (K. S. D.); and University of South Florida (N. G. P.).

This research was funded by National Institute of Mental Health Grant 1 R01 MH63783-Personality Features in Social Deviancy (2002-2005)—awarded to Norman G. Poythress, Jr. We acknowledge and appreciate the assistance and cooperation of the following agencies in collecting data for this research; however, none of the opinions or conclusions expressed in this article reflect any official policy or position of any of these institutions: Drug Abuse Comprehensive Coordinating Office (DACCO), Tampa, Florida; Florida Department of Corrections; Gateway Foundation, Huntsville, Texas; Nevada Department of Prisons; Odyssey House, Salt Lake City, Utah; Operation PAR, Pinellas Park, Florida; Oregon Department of Corrections; Texas Department of Criminal Justice-Institutional Division; Utah Department of Corrections; Volunteers of America, Portland, Oregon; and WestCare, Harris Springs, Nevada.

An earlier version of this research was presented at the 2014 meeting of the American Psychology-Law Society, New Orleans, Louisiana.

Address correspondence to Shannon E. Kelley, Department of Psychology, Texas A\&M University, 4235 TAMU, College Station, TX 77843. E-mail: shannonkelley@tamu.edu 
before age 15 (American Psychiatric Association [APA], 2013). CD typically begins in childhood or adolescence and is characterized by a pervasive pattern of problematic behaviors that infringe on the rights of others and violate social norms (e.g., destruction of property, deceitfulness, physical aggression; APA, 2013). Although CD symptoms seem particularly important to the latent construct tapped by ASPD (Edens, Kelley, Lilienfeld, Skeem, \& Douglas, 2014; Walters \& Knight, 2010), the need to assess historical information to make diagnostic determinations creates difficulties for both self-report and clinical interview measures given the potential for evaluee memory lapse or distortion. Even so, interviewers may have access to relevant collateral information (e.g., institutional records) that attenuates this problem by corroborating and supplementing the evaluee's report.

A related concern is that ASPD has fared poorly in studies on the convergence of self-report and clinical interview-based measures of personality pathology (de Ruiter \& Greeven, 2000; Guy, Poythress, Douglas, Skeem, \& Edens, 2008; Whyte, Fox, \& Coxell, 2006). The convergence of self- and interviewbased measures of personality pathology is mostly poor to fair (Whyte et al., 2006), but self-report does not tend to overdiagnose ASPD as it does with most personality disorders (Blackburn, Donnelly, Logan, \& Renwick, 2004; de Ruiter \& Greeven, 2000; Guy et al., 2008). This suggests that certain features inherent to ASPD (e.g., deceptiveness) exert a unique influence on the information garnered by a particular assessment method. Because self-report and clinical interview measures of ASPD are neither perfect nor interchangeable, their relative strengths and weaknesses across situations should be recognized. In particular, the difficulties associated with each method may be especially pronounced in the assessment of historical information concerning CD.

Advantages of self-report instruments include economy, ease of administration, and facilitation of disclosure, because respondents are less likely to underreport socially undesirable behaviors when questions are self-administered rather than posed by an interviewer (Garb, 2007; Tourangeau \& Yan, 2007). However, for antisocial individuals with tendencies to manipulate, deceive, and minimize faults, ability and willingness to accurately self-report deviant behaviors may be compromised (Lilienfeld, 1994; Whyte et al., 2006; cf. Jones \& Miller, 2012). Idiosyncratic item interpretation and dichotomous response formats that fail to distinguish between pervasive patterns and sporadic occurrences have likewise been noted as weaknesses of some self-report measures (Perry, 1992). Interview-based assessments counter these limitations by allowing for flexibility, probing, consideration of nonverbal cues, and, at least in theory, the detection of deception and poor insight by skilled clinicians. Furthermore, clinical interview is frequently accompanied by a file review, providing the opportunity to supplement and corroborate self-report with factual details (although institutional files may inadequately document childhood behaviors). Nonetheless, the alleged objectivity and superiority of interview over self-report has been challenged by several commentators (Blackburn et al., 2004; Edens, Poythress, Lilienfeld, \& Patrick, 2008; Walters, 2006). Clinicians can be unreliable in integrating contextual information (e.g., social environment) when evaluating behavioral symptoms (Kirk \& Hsieh, 2004) and can be primed by a correctional setting to regard individuals' responses 
with undue suspicion compared to evaluations in noncorrectional populations (e.g., Keulen-de-Vos et al., 2011). Moreover, response distortion during the interview itself may affect the assessment of psychopathic and antisocial traits, even when those ratings are supplemented by file review information (Rogers et al., 2002).

Consistent with previous findings (Guy et al., 2008), clinical interview tends to produce more indicators of antecedent childhood misconduct than does self-report, despite the use of similar questions to elicit such information (Zimmerman \& Coryell, 1990). Furthermore, there is some evidence that the performance of clinical interview differs across the range of pathology severity. For instance, previous dimensional analyses have demonstrated that although CD symptom criteria were meaningful indicators of severe antisociality in a community sample of adolescents, these criteria were not as informative in the less severe range of the latent trait (Gelhorn et al., 2009). However, without direct methodological comparison, it is unclear whether this trend is attributable to the method employed or to the diagnostic criteria.

What is needed to address this issue is a means to compare the performance of self-report and clinical interview across the full range of CD pathology at the test and item levels. Dimensional analyses previously have been useful in comparing assessments of personality disorder from different rater perspectives, including selves and informants (e.g., Cooper, Balsis, \& Oltmanns, 2012). In addition to permitting global and item-level comparisons of CD indicators, dimensional scaling of trait severity assists in determining the level at which criteria are most informative. The goals of the present study are to (a) dimensionally compare the performance of self-report and clinical interview approaches in retrospectively detecting CD symptoms and (b) evaluate test level and individual symptom criteria as indicators of CD severity. We hypothesize that self-report is likely to provide more information at the lower end of the continuum compared to clinical interview, because the strengths of self-report (e.g., increased self-disclosure) may be more salient at this trait level and the potential advantages of the clinical interview may be less relevant. Conversely, we expect that clinical interview will provide more information in identifying CD symptoms at more severe levels of the latent trait where deceptive and minimizing tendencies may impede accurate selfassessment. Correspondingly, we expect that self-report and clinical interview will exhibit differential sensitivity to individual symptoms of $C D$, which vary in their underlying characteristics (e.g., truancy vs. physical cruelty).

\section{METHOD}

\section{PARTICIPANTS}

Participants were 1,159 male offenders either incarcerated in prison $(n=579)$ or participating in court-mandated substance-related residential treatment $(n=580)$. They were recruited from state prisons and residential drug treatment sites in Florida, Nevada, Oregon, Texas, and Utah (see Poythress et al., 2010). Inclusion criteria required that participants (a) self-reported racial 
status as Black or White, (b) spoke English, (c) had an estimated IQ screening of at least 70, and (d) were not receiving psychotropic medication for active symptoms of psychosis. Participants ranged from 17 to 59 years of age with a mean age of 30.49 years $(S D=6.56)$. Most participants were White $(64.1 \%$; $33.8 \%$ African American; 2.1\% not indicated), and $8.2 \%$ of participants additionally identified as ethnically Hispanic. Participants who had missing CD data on the SCID-II or PDQ-4 (described later) or failed to complete either measure were dropped from the dataset $(n=147 ; 11.3 \%)$, resulting in a final sample of 1,159 for the present analyses.

At each site, potential participants were randomly selected from lists of individuals who met inclusion criteria. Following a description of the study, informed consent was obtained using procedures approved by university institutional review boards, and an IQ screening test (Ammons \& Ammons, 1962) was administered. Eligible participants then completed an extensive research protocol that included the administration of the SCID-II ASPD module, the PDQ-4 ASPD scale, and additional measures unrelated to the present analysis. The entire protocol took, on average, 4.5 hours to complete (typically over two sessions) and was supplemented with a detailed institutional file review for each participant. Participants were compensated $\$ 20$ at all but one site where reimbursement was prohibited.

\section{MEASURES}

Structured Clinical Interview for DSM-IV Axis II Personality Disorders (SCIDII) CD Module. The CD module of the SCID-II (First, Spitzer, Gibbon, Williams, \& Benjamin, 1997) is a semistructured interview used to assess the 15 $D S M-I V$ symptom criteria for antecedent $\mathrm{CD}$, which remain unchanged in the updated fifth edition of the DSM (APA, 2013). The SCID-II is a widely used diagnostic instrument that demonstrates high levels of interrater reliability for both categorical and dimensional evaluations of ASPD (Maffei et al., 1997), for which 15 of 22 items assess antecedent CD. Clinical psychology graduate students trained by a senior investigator conducted the clinical interview and rated SCID-II items dichotomously as either criterion present or criterion absent on the basis of participant responses and review of institutional files. For simplicity, we refer to this approach as a clinical interview, although it also includes information derived from collateral records.

Personality Disorder Questionnaire-4 (PDQ-4) CD Scale. The PDQ-4 CD Scale (Hyler, 1994) consists of 15 true-false self-report items with content analogous to SCID-II items (i.e., one for each DSM-IV antecedent CD criterion). Previous studies have examined the psychometric properties of the full 22-item ASPD scale and have found satisfactory discriminatory capability in prison (Davison, Leese, \& Taylor, 2001) and psychiatric inpatient and outpatient samples (Fossati et al., 1998). Previous studies have observed adequate diagnostic concordance between the PDQ-4 and the SCID-II ASPD scales ( $\kappa=.49$; Davison et al., 2001), although results for adult and childhood criteria were not separately reported. 


\section{DATA ANALYSIS}

Analyses were conducted to identify and compare the relative severity level (i.e., the underlying level of the trait) at which CD symptom criteria from the two assessment methods were most informative. Two-parameter probit models provided the most appropriate fit to the self-report and clinical interview CD data. These latent variable models assume that items can vary in terms of both their relatedness-or responsiveness—to the latent trait ("discrimination") and the level of severity at which the relatedness reaches its maximum ("threshold"). Discrimination and threshold parameters for each item were used to estimate relative information provided by a particular item across perspectives. Average parameter values were used to indicate relative information provided by the set of items across perspectives.

\section{RESULTS}

First, we tested the data to determine if they were largely unidimensional with minor influences of correlated item errors. Both the self-report item and the interview item for physical fights were eliminated from the analysis because a preceding exploratory factor analysis revealed that each had an initial extraction less than .20. Our initial confirmatory factor analysis resulted in a not positive definite covariance matrix likely due to the presence of linear dependency, which was remedied by removing the two items for forced sex. Thirteen items from each method (26 total) were thus included and analyzed together on a single latent dimension. We made a priori decisions to (a) allow residuals from corresponding items across perspective to correlate, because both ratings involve self-reported information, and (b) allow residuals from consecutive items within perspective to correlate given the potential for ordering effects due to nonrandom item presentation. Once a valid solution was possible, we made one relatively minor modification by allowing the residuals of the self-report runaway item to correlate with those of the clinical interview curfew violation item because this was the only modification index greater than 100 . On the basis of recommended cutoffs for several fit indices (Hu \& Bentler, 1999), we concluded that the data fit the model sufficiently $(\mathrm{CFI}=.94$, TLI $=.93$, RMSEA $=.05)$ and proceeded to conduct analyses via MPLUS7 in a two-parameter probit model.

As indicated by the item parameters in Table 1, differences at the item level across self and clinical interviewer perspectives were observed. CD symptom criteria are listed in ascending order of threshold parameter values for self-report. Analysis of the threshold parameters using a set of Bonferroni corrected $t$ tests $(p=.05 / 13$ comparisons, or $p=.004)$ showed that 9 of 13 were significantly different across self-report and clinical interview (i.e., displayed nonoverlapping confidence intervals). ${ }^{1}$ For all nine of these items, selves tended

1. Differential item functioning (DIF) analysis would mask the variability between assessment approaches that we are interested in detecting by correcting for these mean differences. However, because our analysis defines items together on a single latent dimension, linking and equating is neither required nor relevant. 
TABLE 1. Item Parameters (and Standard Errors) for Self-Report and Clinical Interview Conduct Disorder Symptom Criteria

\begin{tabular}{|c|c|c|c|c|c|c|}
\hline \multirow[b]{2}{*}{ Criterion } & \multicolumn{3}{|c|}{ Self-Report } & \multicolumn{3}{|c|}{$\begin{array}{c}\text { Clinical } \\
\text { Interview }\end{array}$} \\
\hline & DIS (SE) & THR $(S E)$ & FQ & DIS (SE) & THR $(S E)$ & $\mathrm{FQ}$ \\
\hline 1. Curfew Violation & $0.74(0.06)$ & $-0.82(0.08)^{*}$ & 801 & $0.56(0.05)$ & $1.21(0.11)^{*}$ & 295 \\
\hline 2. Theft & $1.03(0.07)$ & $-0.35(0.05)^{*}$ & 705 & $0.81(0.06)$ & $0.07(0.05)^{*}$ & 585 \\
\hline 3. Breaking and Entering & $1.28(0.09)$ & $-0.03(0.04)^{*}$ & 642 & $1.04(0.08)$ & $0.74(0.06)^{*}$ & 392 \\
\hline 4. Lying & $0.61(0.05)$ & $0.20(0.07)^{*}$ & 530 & $0.71(0.05)$ & $0.81(0.08)^{*}$ & 386 \\
\hline 5. Vandalism & $1.05(0.07)$ & $0.21(0.05)^{*}$ & 566 & $0.89(0.06)$ & $0.88(0.07)^{*}$ & 373 \\
\hline 6. Runaway from Home & $0.64(0.05)$ & $0.28(0.07)^{*}$ & 490 & $0.70(0.05)$ & $0.95(0.08)^{*}$ & 348 \\
\hline 7. Truancy & $0.65(0.05)$ & $0.87(0.08)^{*}$ & 360 & $0.61(0.06)$ & $1.86(0.16)^{*}$ & 191 \\
\hline 8. Theft (Confrontation) & $1.22(0.09)$ & $1.01(0.06)^{*}$ & 266 & $1.35(0.13)$ & $1.51(0.08)^{*}$ & 144 \\
\hline 9. Weapon Use & $0.93(0.07)$ & $1.05(0.07)$ & 285 & $0.91(0.07)$ & $1.23(0.08)$ & 261 \\
\hline 10. Physical Cruelty & $1.09(0.08)^{*}$ & $1.07(0.07)^{*}$ & 257 & $0.73(0.07)^{*}$ & $2.15(0.17)^{*}$ & 129 \\
\hline 11. Fire Setting & $0.66(0.06)$ & $1.53(0.12)$ & 254 & $0.53(0.06)$ & $2.51(0.24)$ & 155 \\
\hline 12. Bullying & $0.66(0.06)$ & $1.69(0.13)$ & 201 & $0.78(0.06)$ & $1.26(0.09)$ & 274 \\
\hline 13. Animal Cruelty & $0.60(0.06)$ & $2.38(0.21)$ & 139 & $0.50(0.06)$ & $2.81(0.29)$ & 138 \\
\hline Average & 0.86 & .70 & & .78 & 1.38 & \\
\hline
\end{tabular}

Note. DIS = discrimination; $\mathrm{THR}=$ threshold; $\mathrm{FQ}=$ frequency. ${ }^{*} p<.003$.

to endorse symptomatic behaviors at lower levels of CD severity compared to clinical interviewers. For bullying items, the opposite, although nonsignificant, trend was observed in which clinical interviewers endorsed the symptom as present at relatively less severe levels of CD. These standings are largely driven by endorsement frequencies, with items endorsed at a higher rate displaying lower thresholds. As seen in Table 1, self-report items generally displayed higher endorsement frequencies relative to clinical interview. Bullying was endorsed more frequently on the clinical interview measure, and weapon use and animal cruelty frequencies were essentially equivalent across methods.

Items differed not only across perspective, but also within perspective. Specifically, certain items tended to indicate lower levels of CD severity whereas other items indicated higher levels of CD severity. For example, within selfreport, theft provided maximal information at a relatively low level of CD severity (threshold parameter $=-0.35$ ). On the other hand, animal cruelty provided maximal information at a relatively high level of CD severity (threshold parameter $=2.38$ ). This general trend consistently held across both perspectives. The threshold parameters estimated via the self-report data were significantly correlated with those estimated via the informant-reported data $(r=.78, p<.001, d f=12)$, indicating that the items were ranked quite similarly across these two perspectives. Similarly, the discrimination parameters estimated via the self-report data were significantly correlated with those estimated via the informant-reported data $(r=.80, p<.001, d f=12)$. That is, selves and clinical interviewers tended to "agree" about the symptom criteria level of responsiveness to the latent trait. 
Differences in information at the item level produced an overall trend in differential information across perspective, with self-report and clinical interview providing different degrees of information across the CD spectrum, as gleaned through parameter averages (see Table 1). Findings confirmed our hypotheses. At relatively low levels of CD severity, the self-report approach tended to provide more information about features of CD compared with clinical interview. Meanwhile, at higher levels of the latent trait, the clinical interview approach provided more information about features of CD relative to self-report.

\section{DISCUSSION}

When taken together, our findings provide a dynamic picture of CD measurement. Although both selves and clinical interviewers tended to agree about the relative order of item severity (e.g., curfew violation is less severe than rape), they tended to respond to each item differently depending upon the degree of CD severity present in the examinee. Consistent with our hypotheses, results demonstrated that selves provide useful information at lower levels of CD severity up to a certain degree, after which point self-report becomes less informative. Furthermore, at a higher point the clinical interview approach begins to provide incremental information regarding the latent trait.

Self-report and interview approaches with the same items may produce different results due to variation in participant responses across measures. Adult offenders, particularly at lower CD levels, may show greater disclosure on anonymous inventories relative to interview formats, and offenders at higher levels of CD severity may conceal or minimize more egregious acts on self-reports. Alternatively, offenders may provide consistent information across measures, with discrepancies reflecting the role of the clinical interviewer in making ultimate determinations. This scenario is consistent with the generally higher endorsement frequencies (and thus lower threshold parameters) observed for self-report relative to clinical interview, which could stem from the absence of a definitive threshold on the self-report measure and offenders' subjective interpretations of what qualifies as a "yes" response. Whereas potentially inappropriate endorsements are final on the questionnaire, a clinical interviewer is able to probe for additional information and clarification, review file data, and formulate judgments of CD symptomatology using contextual information and a standard threshold of behavioral severity/frequency. Indeed, our observed frequency trends resonate with previous research on the PDQ-4, suggesting that the measure could be useful as a brief screening device (Davison et al., 2001).

Given our results, it would be beneficial to develop a system to account for multimethod assessment perspectives. Latent variable models offer one possible approach to integrating the differential information obtained from self-report and clinical interview in such a way that diagnostic determinations could capitalize on each method's contextual strengths. Future research should develop a mechanical means of combining multiple sources of information 
to maximize the information gleaned when assessing CD in various contexts. Where the threshold for categorical diagnoses of CD exists along the continuum, classification accuracy (e.g., false positives), and utility in predicting conceptually relevant outcomes are likewise interesting comparisons between approaches that merit investigation beyond the scope of this study.

Limitations of the present study should be noted. First, the PDQ-4 represents but one of many available self-report measures of $\mathrm{CD}$ and consists of one true-false item for each CD criterion. The generalizability of our findings to other self-report measures requires further study. A related limitation is that items for physical fighting and forced sex were omitted from the study, warranting future attention to the sources of their statistical deficiencies and their potential relevance to multimethod dimensional assessments of CD. Participants were also assured confidentiality, and therefore it is unclear how results might differ in clinical and forensic settings. In particular, because relatively little objective information is likely to be available in institutional files regarding childhood misconduct, both self-report and interview assessment may be based primarily on the evaluee's subjective information. In such applied settings where undesirable consequences of evaluation exist, the role of impression management merits further study, particularly in relation to age. Younger offenders may be more optimistic about deceiving evaluators or, conversely, they may be more truthful than older offenders (perhaps due to inexperience), with either disposition influencing the utility of self-reported information. Although our sample included a wide range of ages, the confidential and retrospective nature of the study precludes reliable analysis of this potential association.

An additional limitation is that our sample consisted exclusively of White and Black adult male offenders. Results in populations of adolescents reporting symptoms concurrently as well as in racially diverse and adult female populations may not align with those from the current study. For youth, objective data regarding childhood antisocial behavior could be more readily available, particularly in juvenile justice settings that might have more accurate or detailed file information concerning CD characteristics. Thus, the incremental utility of self-report regarding antisocial behaviors may be diminished regardless of the level of latent CD severity. Future research should also examine the extent to which our results generalize to community settings where the distribution of CD severity differs. Lastly, data were collected from multiple states and different types of settings; thus the equivalence of our results across these heterogeneous contexts is an important question for future investigation.

Overall, results from this study suggest that self-report and interview methods of CD assessment each contribute information incrementally, albeit at different severity levels of the latent construct. Consideration of the expected severity of CD within a specific population could inform choice of assessment method, although replication is needed to provide further support for our findings and various interpretations. Nonetheless, our study also provides a foundation for developing a statistical means of integrating multimethod assessments in order to optimally measure symptoms of CD and reach accurate diagnostic determinations. 


\section{REFERENCES}

American Psychiatric Association. (2013). Diagnostic and statistical manual of mental disorders (5th ed.). Washington, DC: Author.

Ammons, R. B., \& Ammons, C. H. (1962). The Quick Test (QT): Provisional manual. Psychological Reports, 11, 111-161.

Black, D. W., Gunter, T., Loveless, P., Allen, J., \& Sieleni, B. (2010). Antisocial personality disorder in incarcerated offenders: Psychiatric comorbidity and quality of life. Annals of Clinical Psychiatry, 22(2), 113-120.

Blackburn, R., Donnelly, J. P., Logan, C., \& Renwick, S. J. (2004). Convergent and discriminative validity of interview and questionnaire measures of personality disorder in mentally disordered offenders. Journal of Personality Disorders, 18(2), 129-150.

Cooper, L. D., Balsis, S., \& Oltmanns, T. F. (2012). Self- and informant-reported perspectives on symptoms of narcissistic personality disorder. Personality Disorders: Theory, Research, and Treatment, 3(2), 140-154.

Davison, S., Leese, M., \& Taylor, P. J. (2001). Examination of the screening properties of the Personality Diagnostic Questionnaire 4+ (PDQ-4+) in a prison population. Journal of Personality Disorders, 15(2), 180-194.

de Ruiter, C., \& Greeven, P. (2000). Personality disorders in a Dutch forensic sample: Convergence of interview and self-report measures. Journal of Personality Disorders, 14(2), 162-170.

Edens, J. F., Kelley, S. E., Lilienfeld, S., Skeem, J., \& Douglas, K. (2014). DSM-5 antisocial personality disorder: Predictive validity in a prison sample. Law and Human Behavior. Advance online publication.

Edens, J. F., Poythress, N. G., Lilienfeld, S. O., \& Patrick, C. J. (2008). A prospective comparison of two measures of psychopathy in the prediction of institutional misconduct. Behavioral Sciences of the Law, 26(5), 529-541.

First, M. B., Gibbon, M., Spitzer, R. L., Williams, J. B., \& Benjamin, L. S. (1997). User's guide for the Structured Clinical Interview for DSM-IV axis II personality disorders (SCID-II). Washington, DC: American Psychiatric Press.

Fossati, A., Maffei, C., Bagnato, M., Donati, D., Donini, M., Fiorilli, M.,.... Ansoldi, M. (1998). Criterion validity of the Personality Diagnostic Questionnaire-4+ (PDQ-4+) in a mixed psychiatric sample. Journal of Personality Disorders, 12(2), 172-178.

Garb, H. N. (2007). Computer-administered interviews and rating scales. Psychological Assessment, 19(1), 4-13. doi:http://dx.doi. org/10.1037/1040-3590.19.1.4
Gelhorn, H., Hartman, C., Sakai, J., MikulichGilbertson, S., Stallings, M., Young, S., . . \& \& Crowley, T. (2009). An item response theory analysis of DSM-IV conduct disorder. Journal of the American Academy of Child and Adolescent Psychiatry, 48(1), 42-50.

Guy, L. S., Poythress, N. G., Douglas, K. S., Skeem, J. L., \& Edens, J. F. (2008). Correspondence between self-report and interview-based assessments of antisocial personality disorder. Psychological Assessment, 20(1), 47-54.

Hu, L., Bentler, P. M. (1999). Cutoff criteria for fit indexes in covariance structure analysis: Conventional criteria versus new alternatives. Structural Equation Modeling, 6, $1-55$.

Hyler, S. E. (1994). Personality Diagnostic Questionnaire-4. New York, NY: New York State Psychiatric Institute.

Jones, S., \& Miller, J. D. (2012). Psychopathic traits and externalizing behaviors: A comparison of self- and informant reports in the statistical prediction of externalizing behaviors. Psychological Assessment, 24(1), 255-260.

Keulen-de-Vos, M., Bernstein, D. P., Clark, L. A., Arntz, A., Lucker, T. P., \& de Spa, E. (2011). Patient versus informant reports of personality disorders in forensic patients. Journal of Forensic Psychiatry \& Psychology, 22(1), $52-71$.

Kirk, S., \& Hsieh, D. (2004). Diagnostic consistency in assessing conduct: An experiment on the effect of social context. American Journal of Orthopsychiatry, 74(1), 43-55.

Lilienfeld, S. O. (1994). Conceptual problems in the assessment of psychopathy. Clinical Psychology Review, 14(1), 17-38. doi:http://dx.doi. org/10.1016/0272-7358(94)90046-9

Maffei, C., Fossati, A., Agostoni, I., Barraco, A., Bagnato, M., Deborah, D., ... \& Petrachi, M. (1997). Interrater reliability and internal consistency of the Structured Clinical Interview for DSM-IV Axis II Personality Disorders (SCID-II), version 2.0. Journal of Personality Disorders, 11(3), 279-284.

Perry, J. C. (1992). Problems and considerations in the valid assessment of personality disorders. American Journal of Psychiatry, 149(12), 1645-1653.

Poythress, N. G., Lilienfeld, S. O., Skeem, J. L., Douglas, K. S., Edens, J. F., Epstein, M., \& Patrick, C. J. (2010). Using the PCL-R to help estimate the validity of two self-report measures of psychopathy with offenders. Assessment, 17, 206-219.

Rogers, R., Vitacco, M. J., Jackson, R. L., Martin, M., Collins, M., \& Sewell, K. W. (2002). Faking psychopathy? An examination of 
response styles with antisocial youth. Journal of Personality Assessment, 78(1), 31-46.

Tourangeau, R., \& Yan, T. (2007). Sensitive questions in surveys. Psychological Bulletin, 133(5), 859-883.

Walters, G. D. (2006). Risk-appraisal versus selfreport in the prediction of criminal justice outcomes: A meta-analysis. Criminal Justice and Behavior, 33(3), 279-304.

Walters, G. D., \& Knight, R. A. (2010). Antisocial personality disorder with and without antecedent childhood conduct disorder: Does it make a difference? Journal of Personality Disorders, 24, 258-271.

Whyte, S., Fox, S., \& Coxell, A. (2006). Reporting of personality disorder symptoms in a forensic inpatient sample: Effects of mode of assessment and response style. Journal of Forensic Psychiatry \& Psychology, 17(3), 431-441.

Zimmerman, M., \& Coryell, W. H. (1990). Diagnosing personality disorders in the community: A comparison of self-report and interview measures. Archives of General Psychiatry, 47(6), 527-531. 